\title{
Role of thromboelastography in clinical emergency trauma patients
}

\author{
Fei Geng*, Xiaoxin Liu, Ping Fang, Haiying Hu, Xia Deng, Wenjuan Wang, Yin Li, Youbei Lai, \\ Xiaoxiao Zhong
}

Department of Transfusion, Qian Foshan Hospital, Jinan, Shandong 250000, China

\begin{abstract}
This study aimed to investigate dynamic changes in thromboelastography (TEG) and evaluate its value for emergency trauma patients. TEG values (R, K, Angle, MA and CI) were detected 1, 3, and 7 days post trauma. Followup prognosis data were recorded among all patients and correlations between TEG changes and prognosis were assessed. The results revealed that the $\mathrm{K}$ and $\mathrm{R}$ volumes of the poor prognosis group were obviously higher than those of the good prognosis group at each time point; while the Angle, MA, and CI volumes of the poor prognosis group were relatively lower than those of the good prognosis group, and showed a little rising tendency. This study suggests that variables measured by TEG were closely associated with prognosis evaluation, and highlighted the dangers of coagulation disorder at early stage. TEG is of benefit in evaluating the function of coagulation in clinical emergency trauma patients.
\end{abstract}

Keywords: emergency trauma, coagulation, TEG, prognosis

\section{INTRODUCTION}

Being one of the most serious, common medical situations faced during emergency treatment, severe trauma is estimated to be the leading cause of death for younger people worldwide ${ }^{[1]}$. Acute trauma patients usually present with uncontrolled hemorrhage and coagulopathy and are susceptible to developing "fatal trigemma" - caused by conditions such as hypothermia and acidosis ${ }^{[2,3]}$. Early diagnosis and rapid treatment of coagulopathy are conducive for hemostatic control or trauma resuscitation ${ }^{[4]}$. Therefore, the management of trauma-induced coagulopathy has significant implications for emergency trauma patients ${ }^{[5]}$. However, associations between different coagulopathy patterns that collected from trauma patients and their prognoses have as yet, not been reported.

*Correspondence to: Fei Geng, Qian Foshan Hospital, Jinan, Shandong 250000, China. Email: zhuqian-20404@163.com.

Conflict of interests: The authors declared no conflict of interests.
Thromboelastography (TEG) provides a dynamic graph for detecting the whole process of coagulation. In 1948, German scientist Dr. Hartert described the first thrombotic elastic graph, which was used to test the overall coagulation function of a single blood sample ${ }^{[6]}$. Since the 1980 s, this has also extended to surgical settings to guide blood component transfusion, evaluate patients' hypercoagulable or hypocoagulable state, and investigate coagulation mechanisms ${ }^{[7]}$. In a nutshell, TEG assesses coagulation factors' activity ( $\mathrm{R}$, coagulation reaction time), fibrin level (K, blood clot generation time; Angle, blood clot generation rate), platelet function (MA, maximum amplitude; the ultimate strength of the clot) and coagulation index (CI). The coagulation indicators from conventional coagulation tests (CCT) are only able to reflect the initial coagulation function, and don't provide information for platelet function, thrombotic strength, and fibrinolytic activity. Besides, the temperature in vitro, $\mathrm{pH}$ value and platelet activity are also different from those found in body homeo- 
stasis, which ultimately fail to give a reliable reading of coagulation function in vivo. Moreover, common assays for platelet count and fibrinogen merely show the numerical value rather than the function status of them ${ }^{[8]}$. However, TEG could reflect coagulation and fibrinolysis level in whole blood, providing a rapid, easy-to-read assessment of coagulation at the patient's bedside with the same sample and reagent, regardless of sample treatment and its initial resource, such as whole blood, plasma, and platelet rich plasma ${ }^{[9]}$. The advantages of TEG in monitoring coagulation function have been increasingly paid attention to by clinicians, and are widely used for organ transplantation surgery, cardiovascular surgery, sepsis, acute cerebral infarction, and blood transfusion guidance ${ }^{[10,11]}$.

In this study, TEG values were taken from 54 emergency trauma patients at different time points, along with their prognosis evaluation. Associations between changes in TEG values and prognosis features were evaluated to assess their clinical potential in the prediction of outcomes for emergency trauma patients.

\section{SUBJECTS AND METHODS}

\section{Subjects}

Trauma patients admitted to the Emergency Department of Qian Foshan Hospital from January 2017 to January 2018 were recruited with the following inclusion criteria: over the age of 18 years, transferred to Intensive Care Unit during $24 \mathrm{~h}$ after injury, wound number not less than 2. Exclusion criteria included congenital hemostatic disorders, a known history of primary liver dysfunction or chronic alcoholic, preexisting anticoagulants(such as heparin and vitamin $\mathrm{K}$ antagonists within 6 months of initial injury), longterm aspirin usage, and previous antithrombin or activated protein $\mathrm{C}$ treatment. Based on these criteria, 54 patients were included in our study. Among them there were 27 traffic accident injuries, 13 high falling injuries, 5 explosive debris injuries, and 9 sharpcut injuries. All patients received routine treatment. In addition, follow-up data were collected for the patient cohort and subdivided into two groups based on clinical prognoses: the good prognosis group (31 cases; without hemorrhage and coagulopathy) and the poor prognosis group (23 cases; hemorrhage and coagulopathy) (Fig.1).

\section{Methods}

TEG variables included R, K, Angle, MA, and CI were measured 1,4 , and 7 days after the initial injury.

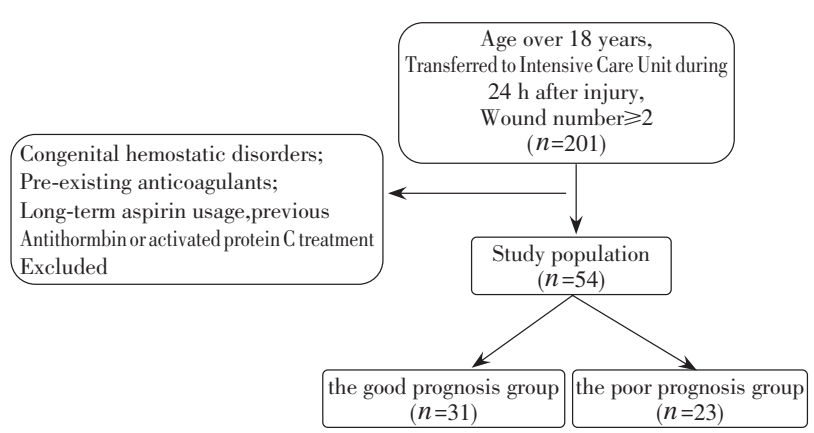

Fig.1 Study population with inclusion and exclusion criteria.

Then dynamic changes in TEG paraments at the different time points between the good and poor prognosis groups were assessed.

\section{Statistical analysis}

All results were presented as mean $\pm \mathrm{SD}$, while all statistical analyses were made using the Graphpad Prism (6.01, La Jolla, CA, USA). Differences between the two groups were analyzed by Student's t test (normal distribution) or Mann-Whitney-Wilcoxon test (non-normal distribution). $P<0.05$ was considered statistically significant.

\section{RESULTS}

\section{Increased $R$ and $K$-time was shown in the poor prognosis group}

As shown in Fig.2A, the R (coagulation reaction time) value from the poor prognosis group was (7.2 $\pm 1.6) \mathrm{min},(6.9 \pm 1.9) \mathrm{min}$ and $(6.8 \pm 1.6) \mathrm{min}$ at day 1 , day 4 and day 7 , respectively, which showed no significant difference between the days. However, the $\mathrm{R}$ value from the good prognosis group displayed a significant downward tendency following an increase value at day 4 . At day 1 and day 7, compared with those of good prognosis group, the $\mathrm{R}$ values were higher in the poor prognosis group.

As shown in Fig.2B, K-time (blood clot generation time) from the good and poor prognosis groups showed the same decline tendency at day 1 , day 4 and day 7. Compared with the poor prognosis group, the good prognosis groups had a significant decreased $\mathrm{K}$ values at day 1 , day 4 and day 7 .

Decreased Angle, MA, CI values as well as a lower rising tendency during day 1 to day 7 in the poor prognosis group

Angle values (blood clot generation rate) from the good and poor prognosis groups showed an upward tendency within 7 days, however, the poor prognosis 
A

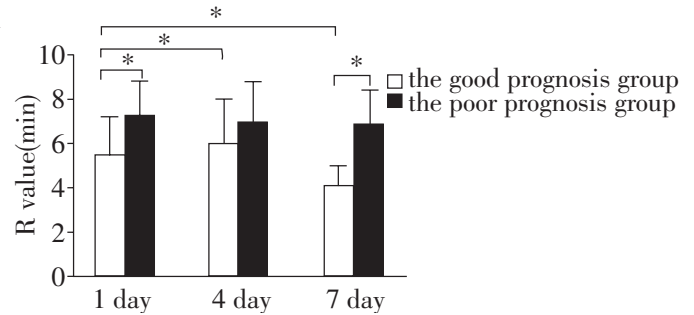

B

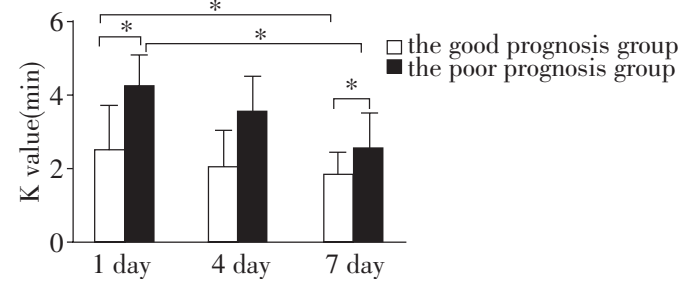

Fig. 2 Change patterns of $R$ and $K$ values from TEG. $R$ value (A) and $K$ value (B) of TEG were collected at day $1,7,14$ from the good prognosis group (31 cases) and the poor prognosis group ( 23 cases) separately. Data are expressed as mean $\mathrm{SD} .{ }^{*} P<0.05$.

group exhibited a decrease at day 7. Compared with the good prognosis group, the poor prognosis group showed decreased Angle values at day 1, day4 and day 7 respectively (Fig.3A).

Compared to the poor prognosis group, the MA values (ultimate strength of clot) from the good prognosis group was more pronounced, with higher values at different time points. Conversely, the poor prognosis group had a relatively higher value at day 4 , but decreased at day 7 (Fig.3B).

The CI (coagulation index) value from the good prognosis group showed a rising tendency over time. A statistically significant difference was reached at day 4 compared to day 1 , however, there was no difference between values at day 4 and day 7 ( $\mathrm{P}>0.05)$. Overall, the patients from the poor prognosis group exhibited a much slower rate of elevation within the 7 days (Fig.3C).

\section{DISCUSSION}

The present study showed that the changing patterns of coagulopathy collected from emergency trauma patients at day 1,4 , and 7 were closely related to their prognosis evaluation. Also, our finding revealed that TEG parameters were of great value in the prediction of emergency trauma outcome.

From the results, we can see that $\mathrm{R}$ and $\mathrm{K}$ values in the good prognosis group were lower than those of the poor prognosis at all time points collected. This suggests that well implemented coagulation corrections at the early time of trauma would be of benefit for the recovery of trauma patients. It should be noted that
A

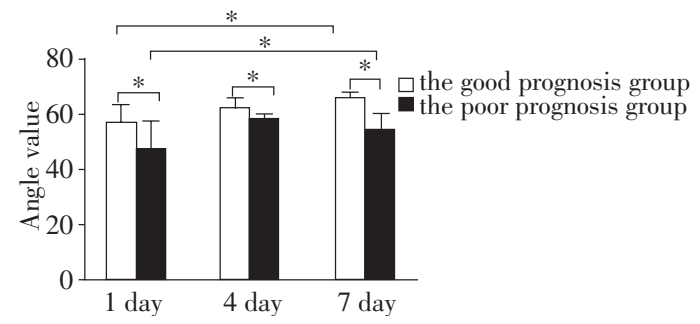

B

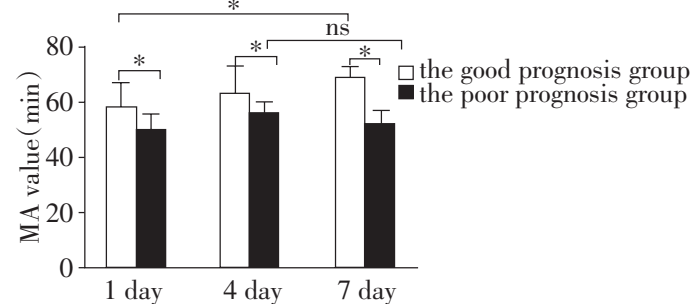

C

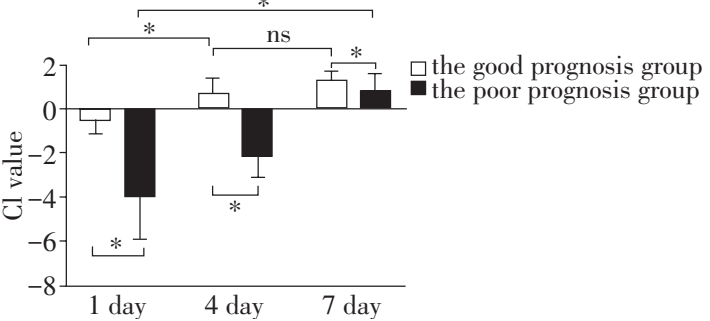

Fig. 3 Change patterns of Angle, MA and CI values from TEG. Angle (A), MA (B) and CI value (C) of TEG were collected at day 1, 7, 14 from the good prognosis group (31 cases) and the poor prognosis group (23 cases) separately. Data are expressed as mean \pm SD. ${ }^{*} P<0.05$.

even though patients from both groups had reduced $\mathrm{R}$ and $\mathrm{K}$ values within 7 days, which means increased coagulation factors and fibrin function in their blood after treatment in hospital, the good prognosis group had a more clearly defined $\mathrm{R}$ and $\mathrm{K}$ recovery value, especially in the first 24 hours. Our results were consistent with a former study, where Nardi et al. showed that an early coagulation support protocol was related to reduced mortality and treatment costs in trauma patients ${ }^{[12]}$. Also, the bad recovery in the poor prognosis group may have been associated with coagulation system dysfunction (within 24 hours) induced infection $^{[13]}$. Therefore, the $\mathrm{R}$ and $\mathrm{K}$ values during the first 24 hours should be seriously monitored in emergency trauma patients considering their important role in the following recovery.

As $\mathrm{K}$ and Angle values are both correlated with fibrin function, our data showed increased Angle values in both the good and poor prognosis groups during patient recovery time, namely in the first 7 days. The patients from the poor prognosis group had a lower Angle value and a more unstable recovery tendency, while patients from the good prognosis group had a gradually increased Angle value. This was concurrent with MA value in the two groups, which indicates 
the change of platelet count or function. This indicated that patients with severe injuries took more time to recover fibrin and platelet function, or were more likely to maintain the hypo-coagulation state based on their delayed propagation of the clot (increased $\mathrm{K}$ and reduced Angle values) and decreased clot strength (reduced MA value). It is reported that platelet impairment is crucial to the development of post injury systemic hyperfibrinolysis in trauma patients ${ }^{[14]}$. Also, a similar change in platelets and leucocyte counts in the first $24 \mathrm{~h}$ after trauma was demonstrated to be associated with the development of septic shock after a week ${ }^{[15]}$. Therefore, keeping an eye on MA value within the first $24 \mathrm{~h}$ as well as its recovery rate for the following days is necessary for the health or prognosis of trauma patients.

The CI value, an index for the comprehensive evaluation of coagulation, from the good prognosis group had a rapid increase from day 1 to 4 , and maintained its value until day 7 . These data suggest that the good prognosis features were closely associated with coagulation function shortly after injury, at least during the first 4 days. Compared to the good prognosis group, the CI values at day 1,4 and 7 of the poor prognosis group all displayed a lower value. The bad coagulation function at day 1 may have accounted for this CI change in the poor prognosis group, even though its value kept increasing during the following days.

Above all, our study investigated the changes of TEG values in emergency trauma patients during the first 7 days after injury and TEG's application in evaluating prognosis. The results showed that patients with a relative hypo-coagulation state, especially during the first 24 hours were less likely to recover after injury, which highlights the need for close observation. Besides, the change rate of TEG values at day 4 and day 7 also provides information for the early treatment time-point. This suggests that an accelerated recovery rate of TEG values will be of benefit for the subsequent or overall recovery of trauma patients. This study was limited, owing to the small sample size, so in accordance all findings need to be verified further. However, as a clinical pilot study, this study offers the latest observation of TEG in a clinical trauma setting, and showed that TEG application is likely to be a good indicator for the outcome of trauma patients.

\section{References}

[1] Kashuk JL, Moore EE, Sawyer M, et al. Postinjury coagulopathy management: goal directed resuscitation via POC thrombelastography. Ann Surg, 2010,
251(4): 604-14.

[2] Roberts HR, Hoffman M, Monroe DM. A cell-based model of thrombin generation. Semin Thromb Hemost, 2006, 32(Suppl 1): 32-8.

[3] Martini WZ, Pusateri AE, Uscilowicz JM, et al. Independent contributions of hypothermia and acidosis to coagulopathy in swine. J Trauma, 2005, 58(5): 1002-9.

[4] Mohamed M, Majeske K, Sachwani GR, et al. The impact of early thromboelastography directed therapy in trauma resuscitation. Scand J Trauma Resusc Emerg Med, 2017, 25(1): 99.

[5] Stensballe J, Henriksen HH, Johansson PI. Early haemorrhage control and management of trauma-induced coagulopathy: the importance of goal-directed therapy. Curr Opin Crit Care, 2017, 23(6): 503-10.

[6] Fischer R, Duraffourd P, Della Santa R. Modes of expression of the thromblastogram; method of Hartert. Praxis, 1954, 43(28): 610-2.

[7] Duffield C, Davies R, Barclay P. Advances in thromboelastograph technology. Anaesthesia, 2018, 73(3): 398-9.

[8] Wade CE, Dubick MA, Blackbourne LH, et al. It is time to assess the utility of thrombelastography in the administration of blood products to the patient with traumatic injuries. J Trauma, 2009, 66(4): 1258.

[9] Leonard SA, Lydon A, Walsh M, et al. Does prior administration of enoxaparin influence the effects of levobupivacaine on blood clotting? Assessment using the Thrombelastograph. Br J Anaesth, 2001, 86(6): 808-13.

[10] Wang SC, Shieh JF, Chang KY, et al. Thromboelastography-guided transfusion decreases intraoperative blood transfusion during orthotopic liver transplantation: randomized clinical trial. Transplant Proc, 2010, 42(7): 2590-3.

[11] Cotton BA, Gunter OL, Isbell J, et al. Damage control hematology: the impact of a trauma exsanguination protocol on survival and blood product utilization. J Trauma, 2008, 64(5): 1177-82.

[12] Nardi G, Agostini V, Rondinelli B, et al. Trauma-induced coagulopathy: impact of the early coagulation support protocol on blood product consumption, mortality and costs. Crit Care, 2015, 19: 83.

[13] Cole E, Davenport R, De'Ath H, et al. Coagulation system changes associated with susceptibility to infection in trauma patients. J Trauma Acute Care Surg, 2013, 74(1): $51-7$.

[14] Moore HB, Moore EE, Chapman MP, et al. Viscoelastic measurements of platelet function, not fibrinogen function, predicts sensitivity to tissue-type plasminogen activator in trauma patients. $J$ Thromb Haemost, 2015, 13(10): 1878-87.

[15] Jol S, Hietbrink F, Leenen LP, et al. Similar change in platelets and leucocytes $24 \mathrm{~h}$ after injury is associated with septic shock a week later. ANZ J Surg, 2017, 87(3): 190-4.

(Received 06 March 2019, Revised 11 April 2019, Accepted 21 April 2019) 